\title{
FLIGHT DELAY PROPAGATION ANALYSIS WITH THE DETAILED POLICY ASSESSMENT TOOL
}

\author{
LISA SCHAEFER and DAVID MILLNER
}

\author{
Center for Advanced Aviation System Development \\ The MITRE Corporation \\ 7515 Colshire Drive, McLean, Virginia, USA 22102-7508
}

\begin{abstract}
The Detailed Policy Assessment Tool (DPAT) models the propagation of delay throughout a system of airports and sectors. We present a DPAT analysis to show the effects of simulated changes in capacity due to inclement weather. Local delays are dependent on the capacity to demand ratio of departures and arrivals. We show propagated effects are significant for the 1st leg after leaving an airport affected by reduced capacities and diminish from leg to leg.
\end{abstract}

\section{Keywords}

Air traffic simulation, delay propagation.

\section{Introduction}

Airline travelers have become familiar in recent years with frequent delays and congestion. Air traffic has become over concentrated at a relatively small number of airports, particularly at certain times of the day. Of the 450 airports handling commercial flights in the USA, the top 60 have 94 percent of the traffic and the top 20 control over half the traffic. Inclement weather in the summers of 1999 and 2000 caused delays not only at airports experiencing the inclement weather, but at airports with flights connecting from the airports experiencing inclement weather [3].

During inclement weather, airport capacity is reduced due to increased aircraft separations. Because instrument landing systems are required for aircraft navigation in these conditions, this situation is called Instrument Meteorological Conditions, or IMC. Clear weather is known as Visual Meteorological Conditions or VMC. DPAT is an air traffic simulation model developed by the MITRE Corporation's Center for Advanced Aviation System Development (CAASD). DPAT simulates the air traffic system as a network of queues. DPAT can be used to analyze how congestion and delays result from the limited capacities of airports and air sectors, and to forecast future congestion to inform policymakers about airport and airspace improvement needs. DPAT also models the propagation of delay throughout a system of airports and sectors. To estimate delays, throughputs, and air traffic congestion in a typical scenario of current operations in the U. S., DPAT models the flow of approximately 50,000 flights per day throughout the airports and airspace of the U. S. National Airspace System (NAS) and can simulate flights to analyze delays at airports around the world.

In the following sections, we present a DPAT analysis to show the effects of simulated changes in capacity and demand for airports in the NAS.

Airspace was not analyzed. We show how reduced capacity due to IMC causes local flight delay and show propagation of delay to other airports. The main contribution of this work is the demonstration and quantitative analysis of propagation and the extent of its effects.

\section{Related Work}

Historical data was used to demonstrate that delay propagates from airport to airport in the NAS. Major airports with a high level of connectivity will experience a large portion of overall system delay, even if the runway conditions at those airports support VMC capacity. As aircraft travel farther along their schedules, the delay is not experienced as greatly at subsequent airports because the effects are damped through airline schedule practices [4].

The FAA [3] describes the increase in delays and cancellations from 1995 through 1999. They found that the current system for collecting causal data does not provide the appropriate data for developing strong conclusions for delay causes and recommend changes to the current data collection system.

Barnhart and Bratu [1] analyze the percentage of passengers connecting at hubs within the context of delays and cancellations. They point out that general delay statistics do not address the issue of passengers missing connecting flights when their flights are delayed arriving to hub airports.

\section{Methodology}

The basic sets of inputs for DPAT relate to air traffic demand and airport capacities. The capacity-todemand ratio at any given time controls how much delays will grow at an airport. In this section we 
describe the data required for this type of analysis and the set of experiments we analyzed.

\section{Required data}

We used the Official Airline Guide (OAG) to develop itineraries for a typical day in year 2000 to represent air traffic demand at airports. The OAG contains all flights the airlines intend to fly. We used an algorithm to connect these flights into sequences of flights, or itineraries. Each simulated aircraft flies to the airports listed in one itinerary. Itineraries are necessary for simulating delay propagation from departure and arrival queues at airports to other airports. General aviation flights were also included since they account for some demand. For more details about DPAT inputs and architecture, see [5].

Queues grow when the number of planes arriving or departing approaches airport capacity. Airport capacities are reduced during inclement weather. When weather clears, the queues start to dissipate because the aircraft waiting in the queue can arrive or depart at a faster rate. Statistics on the queued aircraft are collected after they complete their simulated arrivals or departures.

Airport capacities for VMC were obtained from previous estimates developed by The MITRE Corporation. Capacity curves represent the tradeoff between the number of departures and arrivals an airport can handle, since runways are used for both arrivals and departures. VMC and IMC capacity curves for the three sample airports analyzed in this study, identified here as airports $\mathrm{ABC}, \mathrm{DEF}$, and GHI, are shown in Figs. 1 through 3 [2]. The legend in Fig. 1 shows which curves denote VMC capacities and IMC capacities. VMC capacities represent sustainable levels of operations for good weather conditions. They can be exceeded occasionally. Depending on the runway geometry and visibility, various lower rates can be expected if weather conditions are worse. The IMC curves for this analysis are at approximately $50 \%$ of the VMC capacities, which represent relatively severe capacity reductions relative to VMC. To model IMC at an airport, the arrival and departure capacities were reduced for the duration of the inclement weather.

\section{Experimental scenarios}

Several scenarios were analyzed with DPAT air traffic simulation software. We varied duration of IMC at 5 levels: 15 min., 1 hour, 2 hours, 3 hours, and 4 hours. We ran simulations with only one airport at a time experiencing capacity reductions and one set of runs with all three airports experiencing reduced capacities, for a total of 4 sets of airport IMC combinations.

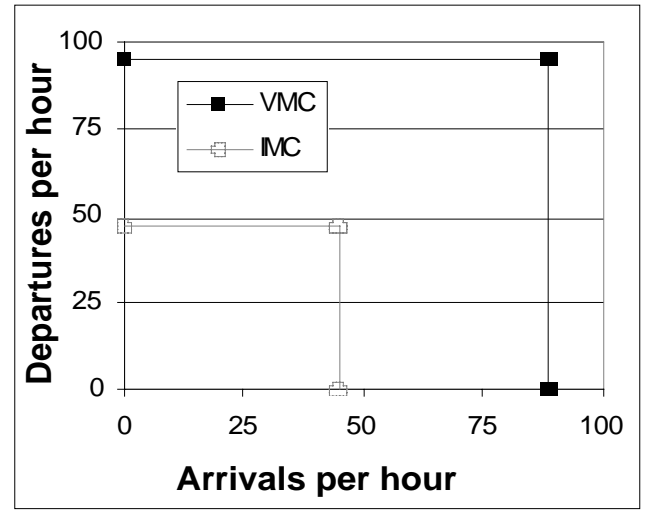

Fig. 1: Arrival and departure capacity tradeoffs for airport $\mathrm{ABC}$ during $\mathrm{VMC}$ and IMC

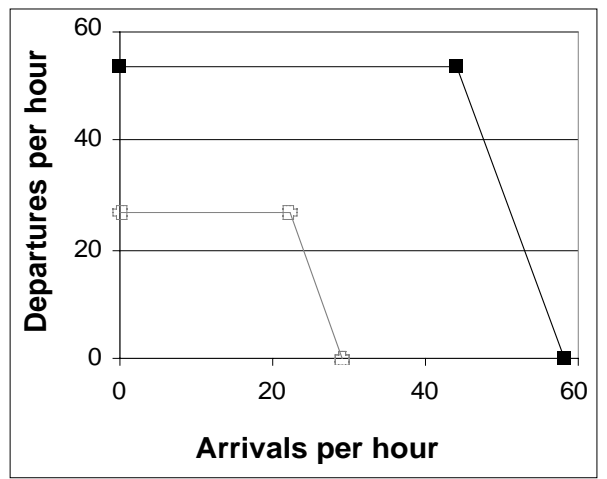

Fig. 2: Arrival and departure capacity tradeoffs for airport DEF during VMC and IMC

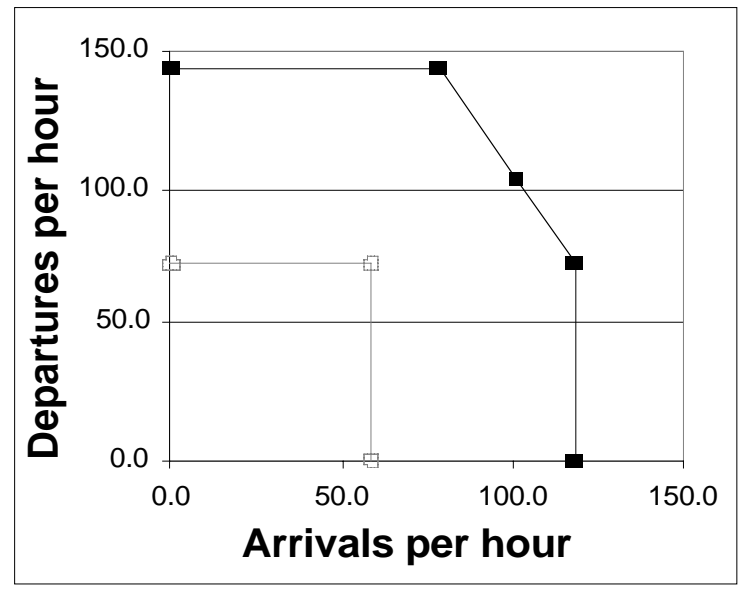

Fig. 3: Arrival and departure capacity tradeoffs for airport GHI during VMC and IMC 
Traffic from approximately 500 other airports was modeled, however we did not analyze these airports individually. Thus we ran 21 scenarios: 5 durations $\mathrm{x}$ 4 IMC combinations, plus 1 scenario with all airports experiencing VMC. These scenarios are hypothetical; and do not represent actual NAS conditions.

All IMC capacity reductions were modeled to occur starting at 9:00 am, during the morning departure rush. After each period of IMC, capacities were set back to VMC capacities and we simulated the remainder of the day to determine the total effects of queueing delay. We analyzed all aircraft scheduled to depart the airport experiencing IMC during an 8 hour period starting at the beginning of IMC capacity reductions and formed a confidence interval for the average amount of delay aircraft experience due to limited capacity during IMC.

\section{Results}

We obtained results for local flight departure and arrival delays due to IMC, propagation for IMC, comparisons to $\mathrm{VMC}$ results, and a comparison of propagated delays to entire system.

Figs. 4 through 7 shows local delay results from 16 DPAT simulations. Fig. 4 contains a legend showing which airport each curve represents. One of the simulation runs used only VMC capacities. The remaining 15 runs had one of three airports experiencing 5 different durations of IMC. Arrival queueing delay is airborne delay accumulated while waiting for the runway at the arrival airport. Departure queueing delay is ground delay accumulated while waiting for a runway at the departure airport. Scheduled departure and arrival delays are relative to the OAG schedule. Each plot shows one type of these four delays for each of the three airports during their respective simulations with IMC at that particular airport.

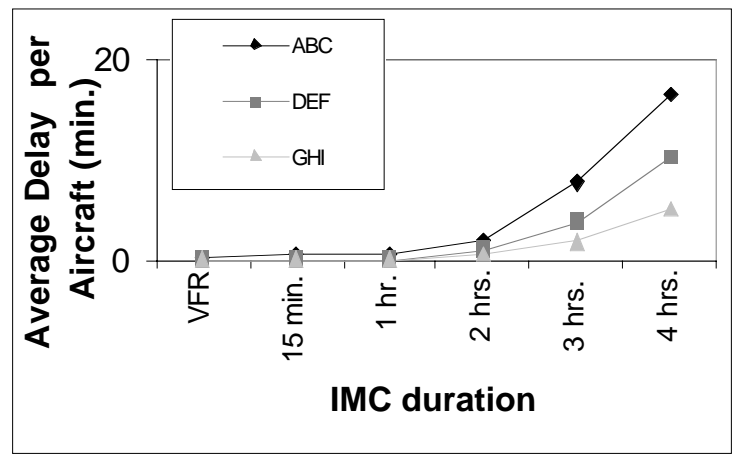

Fig. 4: Average Departure Delay relative to Schedule Departure time at airports $\mathrm{ABC}, \mathrm{DEF}$, and GHI

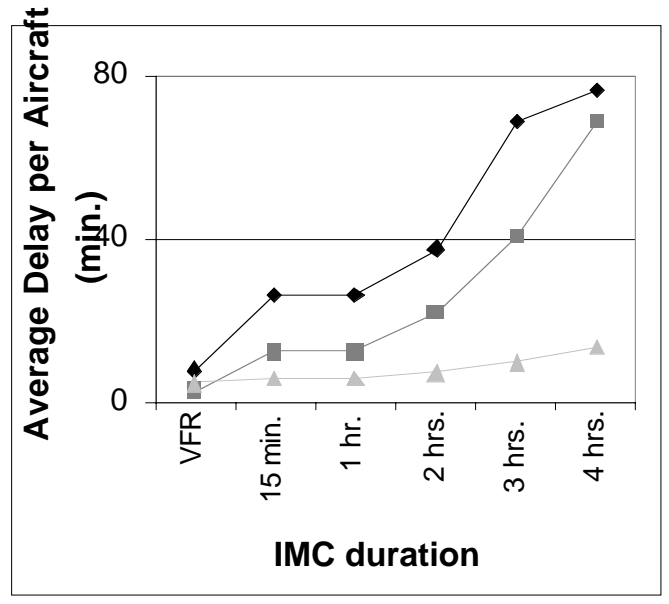

Fig. 5: Average Departure Queueing Delay at airports $\mathrm{ABC}, \mathrm{DEF}$, and GHI

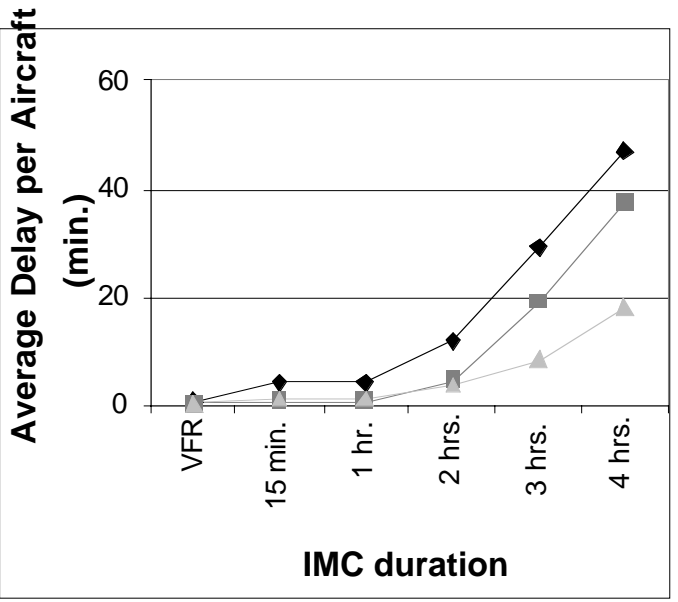

Fig. 6: Average Arrival Delay relative to Schedule Arrival time at airports $\mathrm{ABC}, \mathrm{DEF}$, and $\mathrm{GHI}$

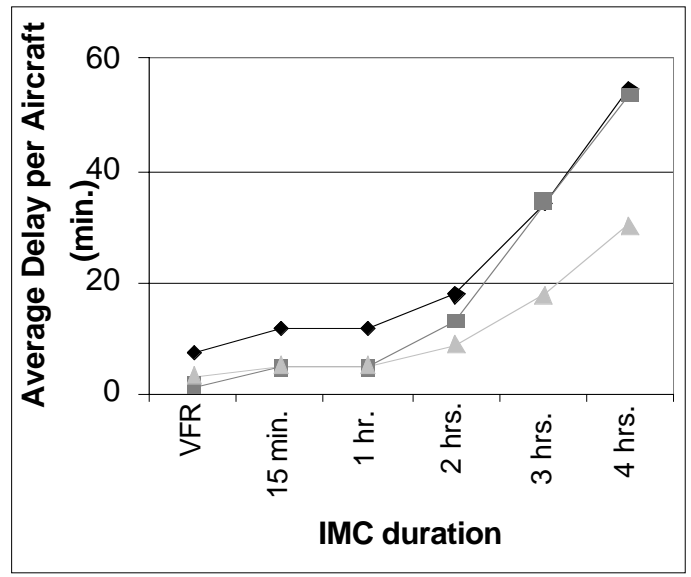

Fig. 7: Average Arrival Queueing Delay at airports $\mathrm{ABC}, \mathrm{DEF}$, and GHI 
Arrival delays are higher than departure delays. Airport GHI consistently experiences lower delays than the other two airports. The capacity to demand ratios for both departures and arrivals airport GHI is consistently higher throughout the analysis period than for the other two airports. The results in Fig. 6 (schedule lateness) are slightly less than the results in Fig. 7 (queueing). The difference between queueing and schedule delays suggests that the schedule is set to consider small delays that may occur randomly within the system. Departure queue delays, Fig. 5, are much higher than departure schedule delays, Fig. 4 , indicating that aircraft were in a large runway queue after leaving the gate.

Figs. 8 through 11 show the $95 \%$ confidence intervals for average scheduled arrival delay for the first through fifth legs of an aircraft itinerary for all flights leaving an IMC airport during the 8 hour period after IMC begins. A legend for the shades used for each duration and labels denoting the order for each leg, $1^{\text {st }}$ through $5^{\text {th }}$, are included on Fig. 8. Twenty treatments were analyzed with the IMC capacity profiles of Figs. 1 through 3: one for each of three airports experiencing IMC conditions for 5 different durations of IMC, plus 5 runs with all three airports experiencing each duration of IMC. 469 flights leave ABC, 249 leave DEF, and 505 leave GHI during the simulation runs with IMC at the respective airports. For the runs with IMC at all three airports, the same number of aircraft left each IMC airport, a total of 1173 flights analyzed for those runs. Aircraft that left two or three of the IMC airports during that time period are counted only once.

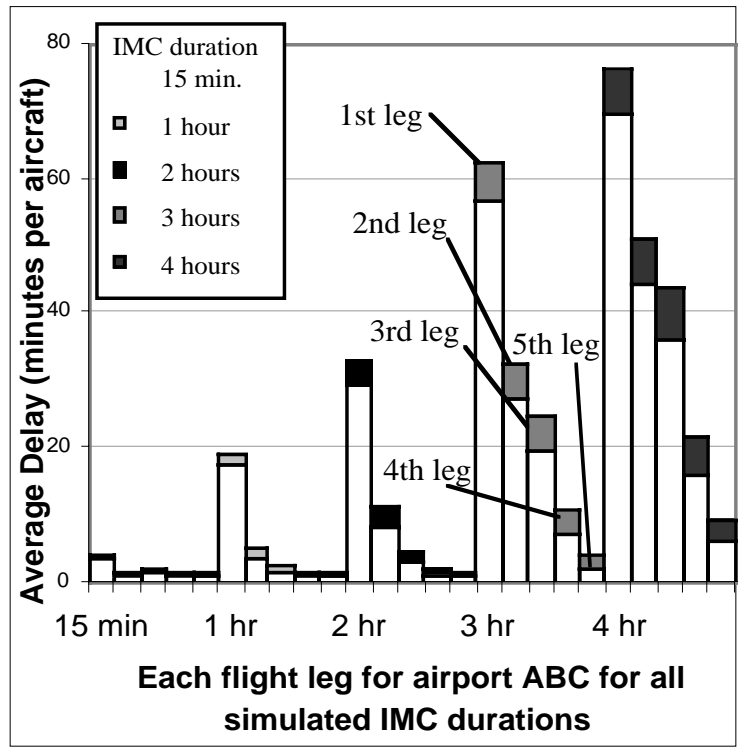

Fig. 8: Confidence intervals for average delay for $1^{\text {st }}$ through $5^{\text {th }}$ flight legs for airport $\mathrm{ABC}$

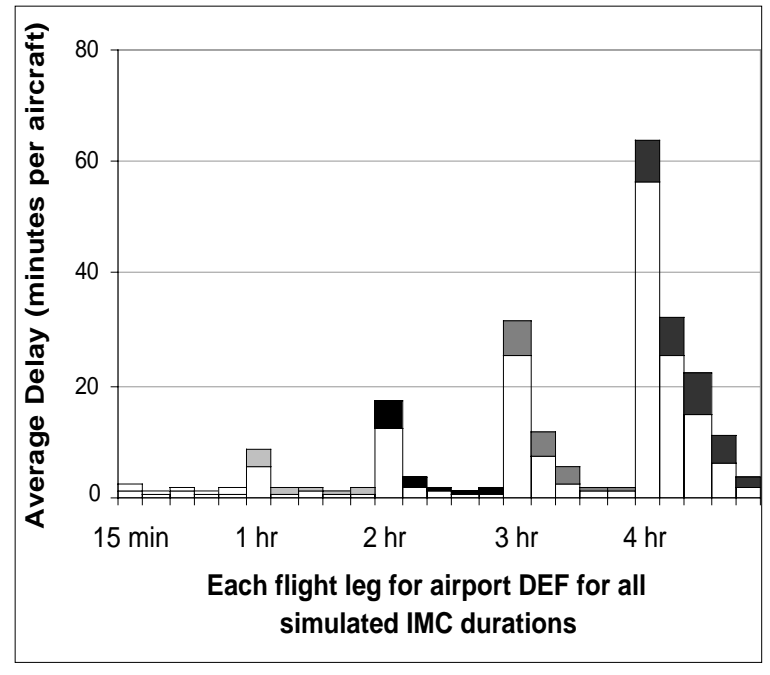

Fig. 9: Confidence intervals for average delay for $1^{\text {st }}$ through $5^{\text {th }}$ flight legs for airport DEF

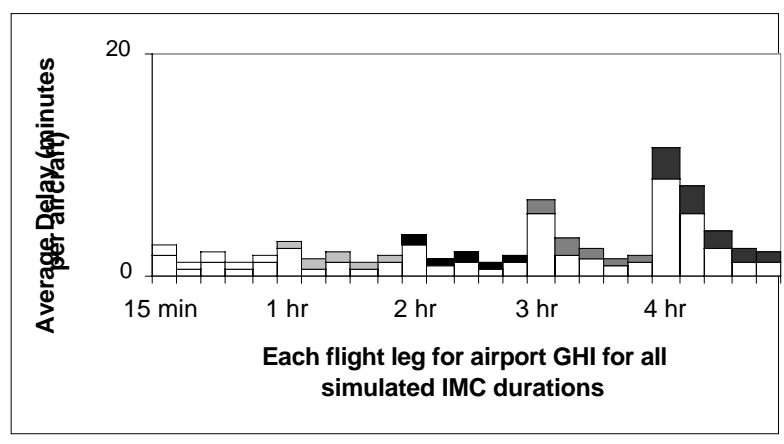

Fig. 10: Confidence intervals for average delay for $1^{\text {st }}$ through $5^{\text {th }}$ flight legs for airport GHI

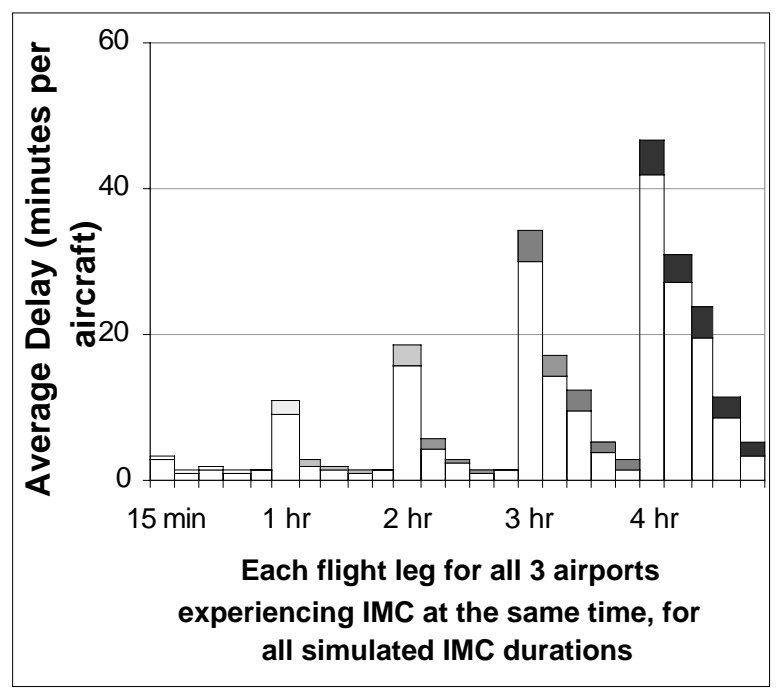

Fig. 11: Confidence intervals for average delay for $1^{\text {st }}$ through $5^{\text {th }}$ flight legs for all 3 airports 
Aircraft leaving airport GHI experience the smallest delays. This is because airport GHI has the highest capacity to demand ratio throughout the 8 hour analysis period. Even though capacities are reduced at airport GHI, the demand is not sufficient to build significant queues.

The confidence intervals for the respective legs for simulations with all 3 airports are slightly higher than an average (not shown) of the confidence intervals for respective legs for the sets of simulations with only one airport at a time experiencing IMC. This is due to the interaction among several airports propagating delays at the same time.

The confidence intervals are binned by IMC duration, represented by different colors. The five bars within each IMC duration bin represent the confidence intervals for the first through fifth legs for the given IMC duration at the given airport. Note that the delay propagated to the first leg is much higher than delay propagated to the second through fifth legs, especially for simulations with 3 or 4 hours of IMC. Delay damps out with each leg suggesting some slack exists in the schedule to allow aircraft to make up for delays.

For VMC, there is no trend across legs, as shown in Fig. 12. When only VMC exists at all airports, delays are not significant, therefore the propagation effect is unobservable. The same set of aircraft analyzed in the IMC runs were analyzed in the simulation with only VMC capacities.

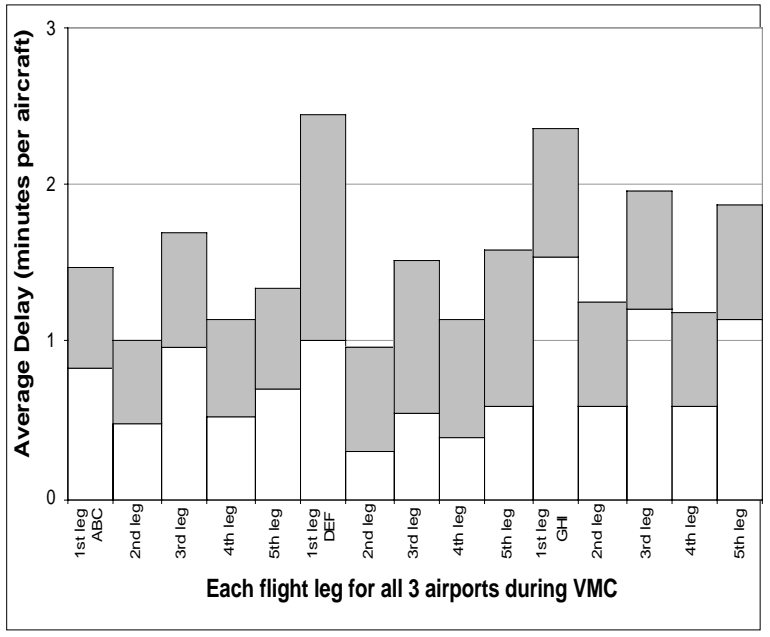

Fig. 12: Delays with VMC significant for the 1st leg after leaving an IMC airport and diminish from leg to leg through the 5th leg. At an airport with a high capacity-to-demand ratio, IMC operations can be accommodated without significantly increasing delay.

\section{Future Work}

This analysis focused on delays incurred due to inclement weather at one airport. When multiple airports are experiencing inclement weather, it would be desirable to develop a method to assign delay to previous airports to determine effects caused by each airport, in addition to accumulating effects from all airports. It would also be interesting to perform an in-depth analysis of the variation in capacity to demand ratios throughout a simulated day and the sensitivity of the results to this ratio.

\section{Acknowledgements}

This work was funded by NavCanada. We would also like to thank Agam Sinha, Ed Jarvis, and Len Wojcik for their comments toward this paper.

\section{References}

1. C. Barnhart and S. Bratu, National Trends in Airline Flight Delays and Cancellations, and the Impact on Passengers, Workshop on Airline and National Strategies for Dealing with Airport and Airspace Congestion, College Park, Maryland, 2001. 2. Federal Aviation Administration, Airport Capacity Benchmark Report 2001, April 2001.

3. Federal Aviation Administration Bureau of Transportation Statistics, Air Carrier Flight Delays and Cancellations, Report no. CR-2000-112, 2000. 4. U.S. House of Representatives. Committee on Appropriations, Subcommittee on Transportation and Related Agencies. Statement of the Federal Aviation Administrator Concerning Air Traffic Control Delays and Capacity. March 15, 2001.

5. F. Wieland, The Detailed Policy Assessment Tool (DPAT) User's Manual, MITRE Technical Report 99W00000012, 1999.

6. F. Wieland, Parallel simulation for aviation applications, Proceedings of the 1998 Winter Simulation Conference, Washington, D.C., 1998.

\section{Conclusions}

When only VMC exists at all airports, delays are not significant, therefore the propagation effect is unobservable. Locally, delay increases with increasing duration of IMC. Propagated effects are 